\title{
Characteristics of Directly Modulated ROF Link for Wireless Access
}

\section{Xavier N. Fernando}

Ryerson University

\author{
Abu B. Sesay \\ University of Calgary
}

digital.library.ryerson.ca/object/98

Please Cite:

Fernando, X. N., \& Sesay, A. B. (2004). Characteristics of directly modulated ROF link for wireless access. Canadian Conference on Electrical and Computer Engineering 2004 (IEEE Cat. No.04CH37513), 4, 2167-2170.

doi:10.1109/CCECE.2004.1347673

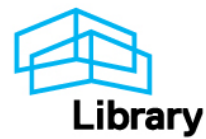




\title{
CHARACTERISTICS OF DIRECTLY MODULATED ROF LINK FOR WIRELESS ACCESS
}

\author{
Xavier N. Fernando and Abu B. Sesay \\ Ryerson University, Toronto, Ontario and University of Calgary, Alberta
}

\begin{abstract}
ABSTRAC'T
The radio-over-fiber (ROF) link is of much interest to provide broadband wireless access. In this paper, we discuss some characteristics of a directly modulated ROF link in CDMA and FDMA environments. These RF measurement results show that, though the ROF link has adequate bandwidth to support several radio channels, nonlinear distortion and power loss, mainly due to electrical to optical conversion process and vice versa, limit the performance. Especially, the AM-PM type nonlinearity that starts at low power levels is a bigger concern. Furthermore, the spurious free dynamic range (SFDR) decreases because the increases with the bandwidth of the RF signal.
\end{abstract}

\section{INTRODUCTION}

Wireless communications has entered a new phase. The focus is shifting from voice to multimedia services. Wireless multimedia access requires wideband radio links. Furthermore, new wireless subscribers are signing up at an increasing rate. These two factors boost the demand for bandwidth.

Micro and pico cellular architecture is an attractive solution. This architecture increases the frequency reuse and capacity. Furthermore, it enables wideband access and saves battery life of portable units. In this pico cell scenario, low power radio access points (RAP) should provide wireless access. Radio-over-fiber (ROF) is the best way to connect these RAPs to the central base station [1]. In this context, the optical carrier is modulated at radio frequencies and transmitted via a single mode fiber that we referred as ROF. In this paper, we discuss characteristics of an ROF based on measurements on a prototype in CDMA and FDMA environments.

The prototype optical transmitter (PFT1700) consists of a directly modulated InGaAsP distributed feedback (DFB) laser transmitter. The receiver (PFR 1701) has a positiveintrinsic-negative (PIN) diode photo detector. These are connected with a 2.2-km long, 9/125 a single mode fiber. FC/APC type connectors are used to reduce the reflection. The receiver has a transimpedance amplifier and a postamplifier with a total gain of $32 \mathrm{~dB}$ to compensate for the

CCECE 2004 - CCGEI 2004, Niagara Falls, May/mai 2004 0-7803$8253-6 / 04 / \$ 17.00$ (C) 2004 IEEE

\begin{tabular}{|l|l|}
\hline Frequency of Operation & 1.7 to $2.2 \mathrm{GHz}$ \\
\hline Wavelength & $1310+/-10 \mathrm{~nm}$ \\
\hline Modulation Gain & $0.12 \mathrm{~mW} / \mathrm{mA}$ \\
\hline DC Optical Responsivity & $0.75 \mathrm{~mA} / \mathrm{mW}$ (min.) \\
\hline Frequency Response Flatness & $+1-0.5 \mathrm{~dB}$ \\
\hline Input 1 dB compression & $1 \mathrm{~dB}$ \\
\hline Input VSWR (50 ohms) & $2: 1$ \\
\hline Third Order Intercept (TOI) & $13 \mathrm{dBm}$ (input) \\
\hline Equivalent Input Noise (max) & $-125 \mathrm{dBm} / \mathrm{Hz}$ \\
\hline Fiber & Single Mode (9/125) \\
\hline
\end{tabular}

Table 1. Key specifications of the optical transmitter receiver pair

$\mathrm{O} / \mathrm{E}$ conversion loss. Therefore, the total link gain is approximately unity at short fiber lengths. Both the low impedance laser and the high impedance detector are reactively matched to a 50-ohm system giving a flat bandpass response from 1.7 to $2.2 \mathrm{GHz}$ and high attenuation elsewhere. The specifications of the transmitter and the receiver are given in Table 1. Unless otherwise specified, all of these are RF measurements taken either at the output of the detector or at the input of the laser. In this paper, 'power' means the RF power into the laser and not the optical power in the fiber.

\section{EXPERIMENTAL SET-UP AND MEASUREMENTS}

Fig. 1 shows the arrangement used to obtain most of the measurements. These experiments were done at TRLabs, Calgary.

\subsection{Noise Measurements}

The characteristics of the noise floor are shown in Fig. 2 where, a single RF tone, at the input and the output of the ROF link is plotted on the same window. These are measured using HP8563 spectrum analyzer and HP8648D RF generator. A strange phenomenon is observed in the noise floor of the ROF link. In Fig. 2, an increment in the noise floor close to center frequency is clearly visible in the figure. 


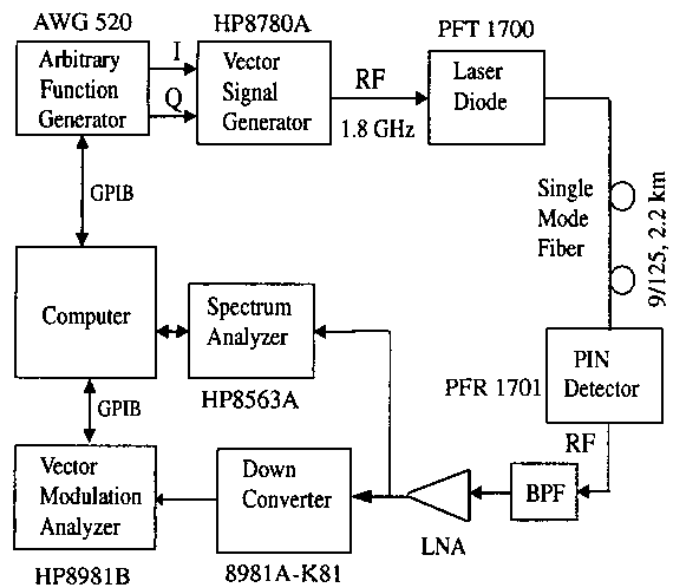

Fig. 1. Experimental setup for dynamic measurements

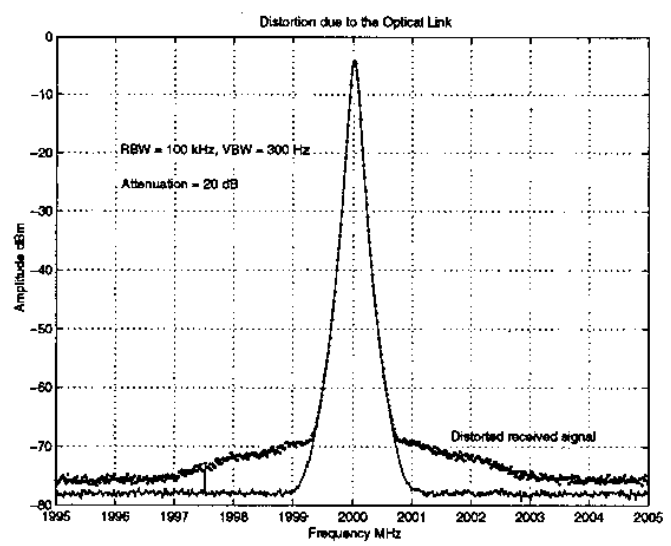

Fig. 2. Distorted noise floor of the ROF link

This frequency dependent increment in the noise floor happens when the input RF power is near the saturation level $(-3 \mathrm{dBm})$. When the carrier power is further increased, this bump in the noise floor further widens and covers the whole spectrum. This phenomena does not occur at low carrier power (less than $-10 \mathrm{dBm}$ ).

\subsection{AM-AM and AM-PM Distortion}

The constellation of vector-modulated symbols plays a key role in the BER performance. The constellations are often distorted due to nonlinear channels. To investigate this effect, Fig. 3 is generated. Here, a random data sequence is generated from the arbitrary waveform generator (AWG520) and an RF carrier is QPSK modulated. This modulated carrier is transmitted via the optical link, received at the remote end, then down converted and the constellation is dis-
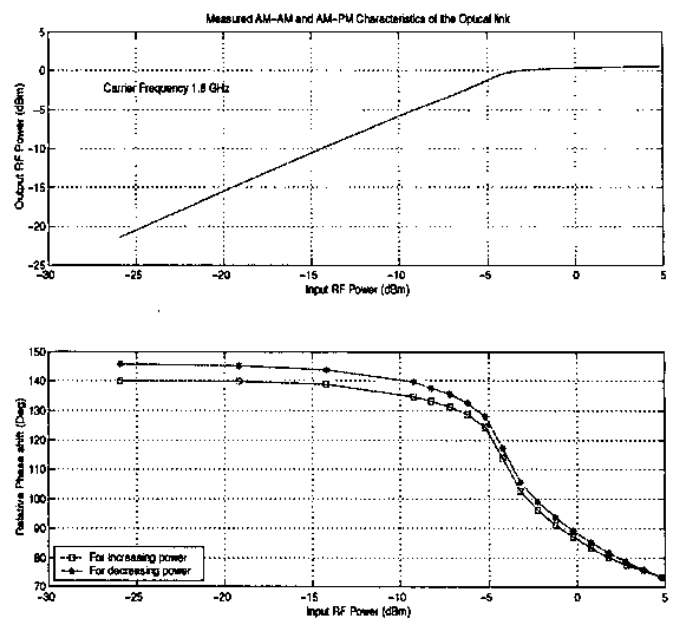

Fig. 3. Amplitude and phase distortions of vector modulated symbols after transmission through the ROF link

played on the vector modulation analyzer. The magnitude and phase of the received vector symbols are measured and this procedure is repeated by changing the input RF power. These are shown in Fig. 3.

From the upper window of the figure, the amplitude of the received constellation starts to saturate at $-3 \mathrm{dBm}$. This is similar to the saturating point at the two-tone tests. The big concern here is the phase shift. The phase of the received QPSK symbols starts to change at a much lower power level $(-15 \mathrm{dBm})$. Furthermore, note that two slightly shifted phase curves are obtained depending on whether the power is increasing or decreasing. During the measurements, the RF power was initially $-24 \mathrm{dBm}$, it was then increased up to $+5 \mathrm{dBm}$ and again decreased to $-24 \mathrm{dBm}$. At the end, there is a residual phase error of $5^{\circ}$. This suggests a hysteresis type, long-term memory of the ROF link [2].

\subsection{Interference Measurements with FDM}

These measurements are taken to analyze the interference between two users with separate RF carriers, that share the same ROF link. Two sets of measurements are done to understand the effect of frequency separation between the users as well as the effect of RF power level of each user.

\subsubsection{Effect of Interfering Power}

First, two independent random data sequences are generated, each at one Mega symbols per second. These are QPSK modulated on to the RF carrier. The carrier frequencies are $1.8 \mathrm{GHz}$ for the desired user and $1.86 \mathrm{GHz}$ for the 
interfering user. Both these carriers are combined and transmitted through the ROF link simultaneously. We found that these frequencies had to be separated by at least $45 \mathrm{MHz}$, due to the limitation of the measuring instruments. (The vector modulation analyzer HP8981B could not distinguish between carriers closer than $45 \mathrm{MHz}$ ).

The interfering power is changed from -10 to $+10 \mathrm{dBm}$ and, each time we decreased the power of the desired user until its constellation is no longer distinguishable. This minimum power of the desired user is recorded. We refer to this power as the 'minimum detectable power' (MDP). When the power is less than this, the BER is very high. Hence, this is a robust way of measuring the BER threshold. This procedure is repeated with and without the optical link and the observations are recorded in Fig. 4. The same experiment is repeated with the interfering frequency $2.1 \mathrm{GHz}$ (frrequency separation $=300 \mathrm{MHz}$ and plotted on the same graph.

From Fig. 4 we observe that, When the carrier separation is $300 \mathrm{MHz}$ or more, there is almost no interference without the optical link. However, with the ROF link, the interfering signal power significantly affects the minimum detectable power (MDP). This phenomenon does not depend on the frequency separation when it is more than 60 $\mathrm{MHz}$.

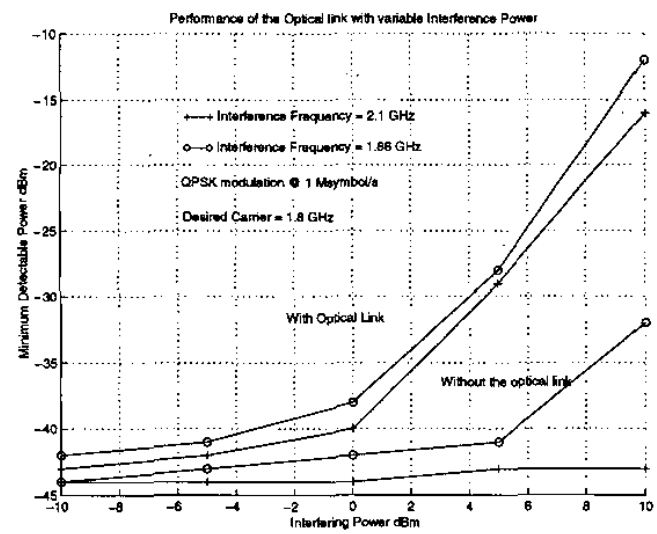

Fig. 4. Effect of the interference power when multiuser signals are transmitted through the ROF link

\subsubsection{Effect of Interfering Frequency}

The above experiment is repeated keeping the interference power constant at $+10 \mathrm{dBm}$ and changing the frequency separation. The observations are plotted in Fig. 5. We observe that, irrespective of the frequency separation, the optical link introduces heavy interference, requiring a MDP of $-15 \mathrm{dBm}$.

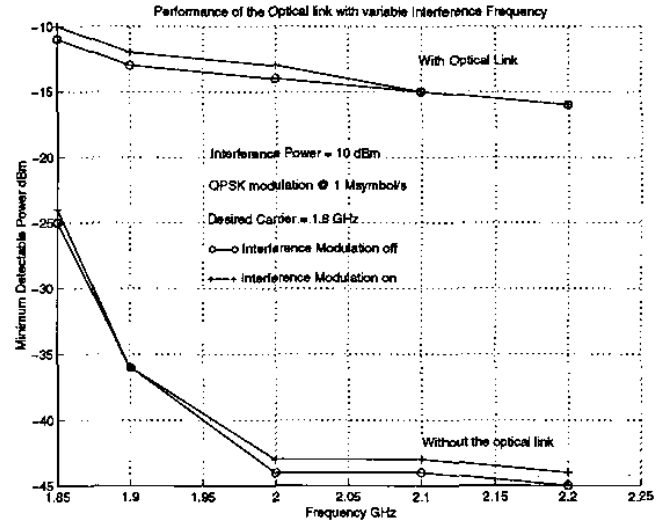

Fig. 5. Effect of the interfering frequency when multiuser signals are transmitted through the ROF link

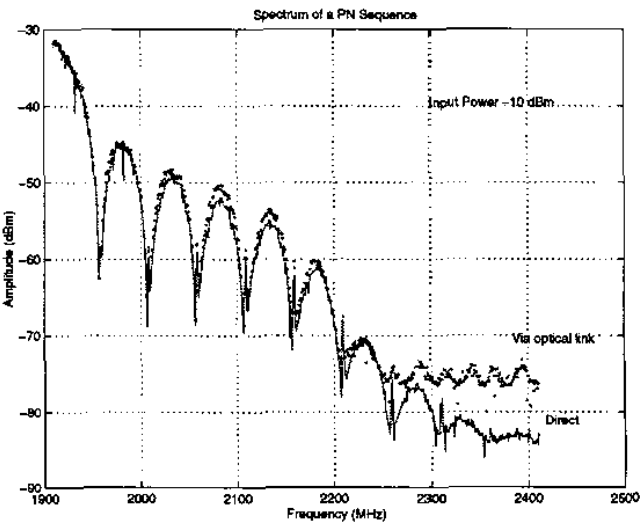

Fig. 6. Power spectral density of a spread spectrum signal after transmission via the ROF link at $-10 \mathrm{dBm}$

From these observations, we conclude that although the optical channel has wide bandwidth, the total power through the link is a big concern. That is, the power of each user through the link dominates interference irrespective to their frequency separation.

\subsection{Spread Spectrum Measurements}

These measurements are made to analyze the deformation in the power density spectrum of a spread spectrum signal through the optical link. First, 1000 bits of random data was generated and it was spread with a PN sequence of length 31 giving 31000 chips. These chips are BPSK modulated on a $1910 \mathrm{MHz}$ RF carrier without any pulse shaping. A chip time $T_{c}$ of $20 n s$ was used. This direct spread signal is transmitted via the ROF link. With this arrangement, the 


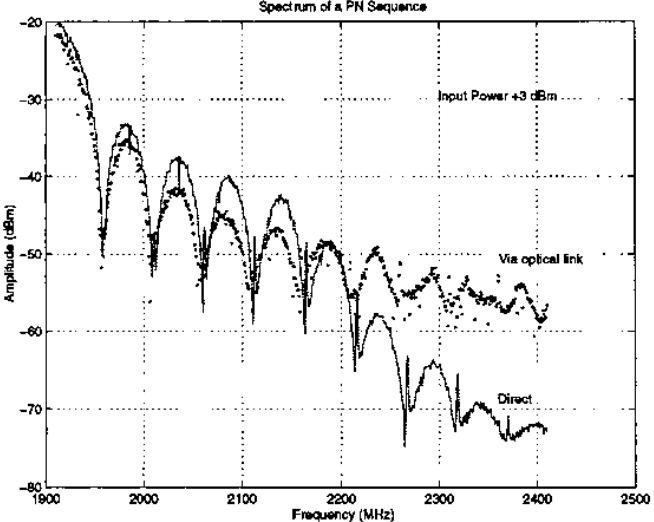

Fig. 7. Power spectral density of a spread spectrum signal after transmission via the ROF link at $+3 \mathrm{dBm}$

transmitted RF power is changed and at each power level, the power spectral density of the received signal is plotted. This procedure is repeated without the optical link for comparison. Both of these data are plotted in the same window in each case. These are shown from Fig. 6 to Fig. 7.

Here, the noise floor is high because of the higher bandwidth and we could detect only up to $-40 \mathrm{dBm}$. This indicates that although the noise floor with single tone was about $-100 \mathrm{dBm}$, with a real spread spectrum signal it is much higher. This significantly reduces the available dynamic range.

As shown in Fig. 6, at the RF power of $-10 \mathrm{dBm}$, both the input spectrum as well as the output spectrum are almost the same up to the sixth null. Beyond the sixth null, the spectrum via optical link shows slightly higher level than the spectrum of the direct signal. Probably this is due to added noise. On Fig. 7 (at $+3 \mathrm{dBm}$ ), energy in the first four sidelobes is less at the output spectrum than the input spectrum. However, at higher (seventh, eighth etc.) side-lobes the distorted spectrum has almost $15 \mathrm{~dB}$ more energy than the direct spectrum. From these, and several other measurements [3], we see that, in general, as the RF power approaches the saturation limit, lower order side-lobes get attenuated while the higher order side-lobes have more power.

\section{DEDUCTIONS FROM THE MEASUREMENTS}

1. The ROF link has adequate bandwidth to support several RF carriers when the total power is within the spurious free dynamic range (SFDR) and the radio frequency is a few $\mathrm{GHz}$. This is true with our prototype link. This also agrees with the theoretical analysis given in [3]

2. The SFDR of the optical link is the most severe con- straint. This will severely limit the cell size and the number of users in a wireless network. The dynamic nonlinear distortions can severely degrade the link performance during sudden power changes.

3. The received power level at the detector is too low, about $-30 \mathrm{dBm}$. This needs to be significantly amplified before driving an antenna $(30-40 \mathrm{~dB})$ in the downlink. Therefore, the nonlinearity (and noise) of the RF amplifiers at the optical receiver introduces further degradation. This is also a big issue in wireless systems.

4. The $-30 \mathrm{dBm}$ received power is only for one channel. If several RF channels are multiplexed, then this power has to be divided among all of those channels. This will need much higher amplifier gain. For instance, if there are $\mathrm{N}$ channels, then the amplifier gain has to be $10 \log (N) \mathrm{dB}$ higher.

5. The AM-PM distortion of the ROF link starts at quite low power level. This should be compensated since all wireless schemes use some form of phase modulation. This is issue is investigated in [4]

6. The noise measurements show a nonlinear phenomena. The noise suddenly increases at the saturation level. This phenomena is further investigated in [5].

\section{REFERENCES}

[1] Winston I. Way, "Optical fiber based microcellular systems: An overview," IEICE Transactions on Communications, vol. E76-B, no. 9, pp. 1091-1102, September 1993.

[2] A. A. M. Saleh, "Frequency independant and frequency dependant nonlinear models of TWT amplifiers," IEEE Transactions on Communications, vol. COM-28, pp. 1715-1720, November 1981.

[3] Xavier Fernando, Signal Processing for Optical Fiber based Fiber Wireless Access, Ph.D. thesis, University of Calgary, 2001.

[4] Xavier Fernando and Abu Sesay, "Adaptive asymmetric linearization of radio over fiber links for wireless access," IEEE Transactions on Vehicular Technology, vol. 51, no. 6, pp. 1576-1586, November 2002.

[5] Xavier Femando, "A stochastic expression for dynamic relative intensity noise," IEEE Photonic Technology Letters, accepted for publication. 